PROCEEDINGS OF THE

AMERICAN MATHEMATICAL SOCIETY

Volume 133, Number 2, Pages 313-320

S 0002-9939(04)07726-3

Article electronically published on September 2, 2004

\title{
AN IMPROVED MORDELL TYPE BOUND FOR EXPONENTIAL SUMS
}

\author{
TODD COCHRANE AND CHRISTOPHER PINNER
}

(Communicated by Wen-Ching Winnie Li)

\begin{abstract}
For a sparse polynomial $f(x)=\sum_{i=1}^{r} a_{i} x^{k_{i}} \in \mathbb{Z}[x]$, with $p \nmid a_{i}$ and $1 \leq k_{1}<\cdots<k_{r}<p-1$, we show that

$$
\left|\sum_{x=1}^{p-1} e^{2 \pi i f(x) / p}\right| \leq 2^{\frac{2}{r}}\left(k_{1} \cdots k_{r}\right)^{\frac{1}{r^{2}}} p^{1-\frac{1}{2 r}},
$$

thus improving upon a bound of Mordell. Analogous results are obtained for Laurent polynomials and for mixed exponential sums.
\end{abstract}

\section{INTRODUCTION}

For a prime $p$, an integer Laurent polynomial

$$
f(x)=a_{1} x^{k_{1}}+\cdots+a_{r} x^{k_{r}}, \quad p \nmid a_{i}, \quad k_{i} \in \mathbb{Z},
$$

where the $k_{i}$ are distinct and nonzero $\bmod (p-1)$, and a multiplicative character $\chi \bmod p$ we consider the mixed exponential sum

$$
S(\chi, f):=\sum_{x=1}^{p-1} \chi(x) e_{p}(f(x))
$$

where $e_{p}(\cdot)$ is the additive character $e_{p}(\cdot)=e^{2 \pi i \cdot / p}$ on the finite field $\mathbb{Z}_{p}$. For $\chi=\chi_{0}$, the principal character, the sum is just a pure exponential sum $S\left(\chi_{0}, f\right)=$ $\sum_{x=1}^{p-1} e_{p}(f(x))$. Of course $S(\chi, f)=0$ unless $\chi^{(p-1) / d}=\chi_{0}$ where $d=\left(k_{1}, \ldots, k_{r}\right.$, $p-1$ ), as is easily seen from the change of variables $x \rightarrow x u$ if there is a $u$ with $u^{d}=1$ and $\chi(u) \neq 1$. The classical Weil bound [12] (see [2] or [10] for Laurent $f$ ),

$$
|S(\chi, f)| \leq d(f) \cdot p^{\frac{1}{2}},
$$

where (assuming for the moment that $k_{1}<\cdots<k_{r}$ )

$$
d(f)= \begin{cases}k_{r}, & \text { if all } k_{i} \text { are positive, } \\ \left|k_{1}\right|, & \text { if all } k_{i} \text { are negative, } \\ \left(k_{r}-k_{1}\right), & \text { otherwise }\end{cases}
$$

is nontrivial only if $d(f)<\sqrt{p}$. The task of improving the Weil bound when $d(f)$ is large remains an open problem. Nontrivial bounds of this kind have only

Received by the editors July 23, 2002 and, in revised form, September 6, 2002.

2000 Mathematics Subject Classification. Primary 11L07, 11L03.

Key words and phrases. Exponential sums.

(C)2004 American Mathematical Society Reverts to public domain 28 years from publication 
been obtained for monomials, binomials and certain sparse polynomials of the type (1.1). Perhaps the most significant result of this type is a bound of Mordell [9] that predates the work of Weil:

$$
|S(\chi, f)| \leq\left(\begin{array}{c}
2 r \\
r
\end{array}\right)^{\frac{1}{2 r}}\left(1-\frac{1}{p}\right)^{\frac{-1}{2 r}}\left(p-1, k_{1}, \ldots, k_{r}\right)^{\frac{1}{2 r}}\left(\ell_{1} \ell_{2} \cdots \ell_{r}\right)^{\frac{1}{2 r}} p^{1-\frac{1}{2 r}},
$$

where

$$
\ell_{i}= \begin{cases}k_{i}, & \text { if } k_{i}>0, \\ r\left|k_{i}\right|, & \text { if } k_{i}<0 .\end{cases}
$$

Although Mordell only considered pure exponential sums, his method easily extends to the case of mixed sums. Davenport 5] obtained some refinements of Mordell's work, but his results were superseded by Weil; see also Shparlinski [11 p.88]. For further discussion of the case of monomials see [6] and [8], and for binomials [7, [1], 3] and [14.

We show here how a simple application of Hölder's inequality (of the type employed by Heath-Brown and Konyagin in [6]) can substantially improve the bound of Mordell:

Theorem 1.1. For any $f$ and $\chi$ as above,

$$
|S(\chi, f)| \leq 2^{\frac{2}{r}}\left(\ell_{1} \cdots \ell_{r}\right)^{\frac{1}{r^{2}}} p^{1-\frac{1}{2 r}} .
$$

The theorem implies a nontrivial bound on $|S(\chi, f)|$ for $\ell_{1} \ell_{2} \ldots \ell_{r}<4^{-r} p^{r / 2}$. The following example shows that in general the exponent $1 / r^{2}$ on the product $\left(\ell_{1} \cdots \ell_{r}\right)$ cannot be improved. In this example, $\left(\ell_{1} \ell_{2} \cdots \ell_{r}\right)^{1 / r^{2}} \sim p^{1 / 2 r} 2^{-\frac{1}{2 r}}(r / 2) !^{\frac{1}{r^{2}}}$ as $p \rightarrow \infty$, while $|S(\chi, f)| \sim p / 2$.

Example 1.1. If $r$ is even, $p>r / 2$ and $f(x)=\sum_{i=1}^{r / 2}\left(x^{\frac{p-1}{2}+i}-x^{i}\right)$, then

$$
\sum_{x=1}^{p-1} e_{p}(f(x))=\frac{p-1}{2}+\sum_{\left(\frac{x}{p}\right)=-1} e_{p}\left(-2\left(x+x^{2}+\cdots+x^{r / 2}\right)\right),
$$

and so by Weil's bound,

$$
\left|S\left(\chi_{0}, f\right)-\frac{p-1}{2}\right| \leq \frac{r}{2} \sqrt{p} .
$$

Weaker types of bounds on $\left|S\left(\chi_{0}, f\right)\right|$, nontrivial even when all the $k_{i}$ are on the order of $p$ in size, were obtained by the authors in 4 .

A key ingredient in the proof of both Mordell's theorem and our own is the estimation of

$$
M=\#\left\{\left(x_{1}, \ldots, x_{r}, y_{1}, \ldots, y_{r}\right) \in \mathbb{Z}_{p}^{* 2 r}: \sum_{i=1}^{r} x_{i}^{k_{j}}=\sum_{i=1}^{r} y_{i}^{k_{j}}, j=1, \ldots, r\right\} .
$$

Mordell deduced (1.3) from the bounds

$$
|S(\chi, f)| \leq\left(1-\frac{1}{p}\right)^{\frac{-1}{2 r}}\left(p-1, k_{1}, \ldots, k_{r}\right)^{\frac{1}{2 r}} p^{\frac{1}{2}-\frac{1}{2 r}} M^{\frac{1}{2 r}}
$$

and

$$
M \leq\left(\begin{array}{c}
2 r \\
r
\end{array}\right) \ell_{1} \ell_{2} \ldots \ell_{r} p^{r}
$$


Here, we prove

Theorem 1.2. For any $f$ and $\chi$ as above,

$$
|S(\chi, f)|<(p-1)^{1-\frac{2}{r}} p^{\frac{1}{2 r}} M^{\frac{1}{r^{2}}} .
$$

Using the bound of Mordell (1.5) and $\left(\begin{array}{c}2 r \\ r\end{array}\right)<2^{2 r}$ one immediately obtains Theorem 1.1. In Lemma 3.1, we obtain a slight refinement of (1.5) using a version of Bezout's Theorem proved by Wooley [13. We also state a sharp upper bound on $M$ for the case $r=2$ in Lemma 3.2.

For $r=1$ and $k_{1}=k$, plainly $M=(k, p-1)(p-1)$, and we recover from Theorem 1.2 the Weil bound for twisted Gauss sums,

$$
\left|\sum_{x=1}^{p-1} \chi(x) e_{p}\left(a x^{k}\right)\right| \leq(k, p-1) \sqrt{p} .
$$

For $r=2,1 \leq l<k$, we obtain from Theorem 1.2 and Lemma 3.2,

$$
\left|\sum_{x=1}^{p-1} \chi(x) e_{p}\left(a x^{k}+b x^{l}\right)\right| \leq(k l)^{1 / 4} p^{3 / 4},
$$

which is nontrivial and improves on Weil (1.2) when $(p l)^{\frac{1}{3}}<k<p / l$, and

$$
\left|\sum_{x=1}^{p-1} \chi(x) e_{p}\left(a x^{k}+b x^{-l}\right)\right| \leq(3 k l)^{1 / 4} p^{3 / 4} \text {. }
$$

\section{Proof of Theorem 1.2}

For $\vec{u}=\left(u_{1}, \ldots, u_{r}\right) \in \mathbb{Z}_{p}^{r}$, we define

$$
N(\vec{u})=\#\left\{\left(x_{1}, \ldots, x_{r}\right) \in \mathbb{Z}_{p}^{* r}: \sum_{i=1}^{r} x_{i}^{k_{j}}=u_{j}, j=1, \ldots, r\right\}
$$

and observe that

$$
\sum_{\vec{u} \in \mathbb{Z}_{p}^{r}} N(\vec{u})=(p-1)^{r}, \quad \sum_{\vec{u} \in \mathbb{Z}_{p}^{r}} N^{2}(\vec{u})=M .
$$

For any multiplicative character $\chi$,

$$
\begin{aligned}
& \sum_{\vec{u} \in \mathbb{Z}_{p}^{r}}\left|\sum_{m=1}^{p-1} \chi^{r}(m) e_{p}\left(a_{1} u_{1} m^{k_{1}}+\cdots+a_{r} u_{r} m^{k_{r}}\right)\right|^{2 r} \\
& =\sum_{\substack{x_{1}, \ldots, x_{r}, y_{1}, \ldots, y_{r} \in \mathbb{Z}_{p}^{*}}} \chi^{r}\left(x_{1} \ldots x_{r} y_{1}^{-1} \ldots y_{r}^{-1}\right) \sum_{\vec{u} \in \mathbb{Z}_{p}^{r}} e_{p}\left(\sum_{j=1}^{r} a_{j} u_{j}\left(x_{1}^{k_{j}}+\cdots+x_{r}^{k_{j}}-y_{1}^{k_{j}} \cdots-y_{r}^{k_{j}}\right)\right) \\
& =p^{r} \sum^{*} \chi^{r}\left(x_{1} \ldots x_{r} y_{1}^{-1} \ldots y_{r}^{-1}\right) \leq p^{r} M,
\end{aligned}
$$

where $\sum^{*}$ denotes a sum over the $x_{1}, \ldots, x_{r}, y_{1}, \ldots, y_{r}$ in $\mathbb{Z}_{p}^{*}$ satisfying $\sum_{j=1}^{r} x_{j}^{k_{i}}=$ $\sum_{j=1}^{r} y_{j}^{k_{i}}$ for $1 \leq i \leq r$. 
Writing $S=S(\chi, f)$, we have

$$
\begin{aligned}
(p-1) S^{r} & =\sum_{m=1}^{p-1}\left(\sum_{x=1}^{p-1} \chi(m x) e_{p}\left(a_{1}(m x)^{k_{1}}+\cdots+a_{r}(m x)^{k_{r}}\right)\right)^{r} \\
& =\sum_{m=1}^{p-1} \chi^{r}(m) \sum_{x_{1}, \ldots, x_{r} \in \mathbb{Z}_{p}^{*}} \chi\left(x_{1} \ldots x_{r}\right) e_{p}\left(\sum_{j=1}^{r} a_{j} m^{k_{j}}\left(x_{1}^{k_{j}}+\cdots+x_{r}^{k_{j}}\right)\right) \\
& =\sum_{x_{1}, \ldots, x_{r} \in \mathbb{Z}_{p}^{*}} \chi\left(x_{1} \ldots x_{r}\right) \sum_{m=1}^{p-1} \chi^{r}(m) e_{p}\left(\sum_{j=1}^{r} a_{j} m^{k_{j}}\left(x_{1}^{k_{j}}+\cdots+x_{r}^{k_{j}}\right)\right),
\end{aligned}
$$

and so

$$
(p-1)|S|^{r} \leq \sum_{\vec{u} \in \mathbb{Z}_{p}^{r}} N(\vec{u})\left|\sum_{m=1}^{p-1} \chi^{r}(m) e_{p}\left(\sum_{j=1}^{r} a_{j} u_{j} m^{k_{j}}\right)\right| .
$$

Applying Hölder's inequality twice, the second time splitting

$$
N(\vec{u})^{\frac{2 r}{2 r-1}}=N(\vec{u})^{\frac{2 r-2}{2 r-1}} N(\vec{u})^{\frac{2}{2 r-1}},
$$

and using (2.1) and (2.2) gives

$$
\begin{aligned}
(p-1)|S|^{r} \leq & \left(\sum_{\vec{u}} N(\vec{u})^{\frac{2 r}{2 r-1}}\right)^{\frac{2 r-1}{2 r}} \\
& \times\left(\sum_{\vec{u}}\left|\sum_{m=1}^{p-1} \chi^{r}(m) e_{p}\left(a_{1} u_{1} m^{k_{1}}+\cdots+a_{r} u_{r} m^{k_{r}}\right)\right|^{2 r}\right)^{\frac{1}{2 r}} \\
\leq & \left(\left(\sum_{\vec{u}} N(\vec{u})\right)^{\frac{2 r-2}{2 r-1}}\left(\sum_{\vec{u}} N^{2}(\vec{u})\right)^{\frac{1}{2 r-1}}\right)^{\frac{2 r-1}{2 r}}\left(M p^{r}\right)^{\frac{1}{2 r}} \\
= & \left((p-1)^{r}\right)^{\frac{r-1}{r}}(M)^{\frac{1}{2 r}}\left(M p^{r}\right)^{\frac{1}{2 r}}=(p-1)^{r-1} p^{\frac{1}{2}} M^{\frac{1}{r}} .
\end{aligned}
$$

Hence

$$
|S|<(p-1)^{1-\frac{2}{r}} p^{\frac{1}{2 r}} M^{\frac{1}{r^{2}}} .
$$

\section{Estimation of $M$}

We establish here the following refinement of Mordell's upper bound (1.5).

Lemma 3.1. For any integers $k_{i}, 1 \leq i \leq r$, distinct and nonzero $\bmod (p-1)$,

$$
M \leq \frac{4 e}{r^{2}}\left(\begin{array}{c}
2 r \\
r
\end{array}\right)\left(\ell_{1} \cdots \ell_{r}\right)(p-1)^{r},
$$

where the $e=2.718 \ldots$ can be dropped if all the exponents are positive.

The factor of $e$ can also be removed when $r=3$, while for $r=2$ we have the sharper Lemma 3.2. If all $k_{i}$ are positive, it is reasonable to conjecture

$$
M \leq k_{1} k_{2} \ldots k_{r} p^{r}
$$

which would be best possible in view of the following example. 
Example 3.1. Let $k \mid(p-1)$ be a fixed positive integer, and suppose that $k_{i}=k \cdot i$, $1 \leq i \leq r$ and $p>r$. Then for any choice of $y_{1}, y_{2}, \ldots, y_{r}$, it follows from the Newton-Girard identities (see e.g. Mordell [9]) that for any solution $x_{1}, x_{2}, \ldots, x_{r}$ satisfying the defining system for $M$, the $r$-tuple $\left(x_{1}^{k}, x_{2}^{k}, \ldots, x_{r}^{k}\right)$ is just a permutation of the $r$-tuple $\left(y_{1}^{k}, y_{2}^{k}, \ldots, y_{r}^{k}\right)$. Conversely, any such $x_{1}, \ldots, x_{r}$ is trivially a solution. Hence we obtain

$$
M \sim k_{1} k_{2} \cdots k_{r} p^{r}, \quad \text { as } p \rightarrow \infty .
$$

For the case $r=2$ we obtain this best possible estimate.

Lemma 3.2. If $r=2$, then we have

$$
M< \begin{cases}k_{1} k_{2}(p-1)^{2}, & \text { if } 1 \leq k_{1}<k_{2}, \\ 3\left|k_{1}\right| k_{2}(p-1)^{2}, & \text { if } k_{1}<0<k_{2} .\end{cases}
$$

For a positive integer $l \mid \frac{1}{2}(p-1)$ and exponents $k_{1}=l, k_{2}=2 l$ or $k_{1}=-l, k_{2}=l$ it is readily verified that $M=2 l^{2}(p-1)^{2}-l^{3}(p-1)$ and $M=3 l^{2}(p-1)^{2}-3 l^{3}(p-1)$ respectively, so the constants in both these bounds are sharp. If $r>2$ and some of the $k_{i}$ are negative, then it is unclear to the authors what the best possible upper bound should be, although the example $k_{1}, k_{2}=-l, l$ just noted shows that one cannot get $M<\ell_{1} \ldots \ell_{r} p^{r}$ in general.

Proof of Lemma 3.1. Order the $k_{i}$ in terms of increasing $\ell_{i}$,

$$
\ell_{1} \leq \ell_{2} \leq \cdots \leq \ell_{r} .
$$

Let $S$ denote the set of solutions to be counted:

$$
S=\left\{(\vec{x}, \vec{y}): \vec{x}=\left(x_{1}, \ldots, x_{r}\right), \vec{y}=\left(y_{1}, \ldots, y_{r}\right) \in \mathbb{Z}_{p}^{* r}, \sum_{j=1}^{r} x_{j}^{k_{i}}=\sum_{j=1}^{r} y_{j}^{k_{i}}, i=1, \ldots, r\right\} .
$$

For $d \leq r$ write

$$
D_{d}\left(u_{1}, \ldots, u_{d}\right)=\operatorname{det}\left(u_{i}^{k_{j}}\right)_{1 \leq i, j \leq d} .
$$

A result of Wooley [13] shows that for any set of $\alpha_{1}, \ldots, \alpha_{d} \in \mathbb{Z}_{p}$,

$$
\#\left\{\vec{u}=\left(u_{1}, \ldots, u_{d}\right) \in \mathbb{Z}_{p}^{* d}: D_{d}(\vec{u}) \neq 0, \sum_{j=1}^{d} u_{j}^{k_{i}}=\alpha_{i}, \quad i=1, . ., d\right\} \leq \ell_{1} \cdots \ell_{d}
$$

(since we are solving the simultaneous polynomial congruences $F_{i}\left(u_{1}, \ldots, u_{d}\right)=0$, $i=1, \ldots, d$, with the degree $k_{i}$ polynomial $F_{i}=\sum_{j=1}^{d} u_{j}^{k_{i}}-\alpha_{i}$ when $k_{i}>0$ and the degree (at most) $d\left|k_{i}\right|$ polynomial $F_{i}=u_{1}^{\left|k_{i}\right|} \cdots u_{d}^{\left|k_{i}\right|}\left(\alpha_{i}-\sum_{j=1}^{d} u_{j}^{k_{i}}\right)$ when $k_{i}<0$; it is readily checked that the non-vanishing of $\operatorname{det}\left(\frac{\partial F_{i}}{\partial u_{j}}\right)$ amounts to $\left.D_{d}\left(u_{1}, \ldots, u_{d}\right) \neq 0\right)$.

For a vector $\vec{u}=\left(u_{1}, \ldots, u_{d}\right) \in \mathbb{Z}_{p}^{* d}$ define

$$
\begin{gathered}
\kappa(\vec{u})=\max \left\{l: D_{l}\left(v_{1}, \ldots, v_{l}\right) \neq 0 \text { for some }\left\{v_{1}, \ldots, v_{l}\right\} \subseteq\left\{u_{1}, \ldots, u_{d}\right\}\right\}, \\
M_{d, l}=\#\{(\vec{x}, \vec{y}) \in S: \kappa(\vec{x})=d, \kappa(\vec{y})=l\},
\end{gathered}
$$

and for $l \leq d$,

$$
N_{d, l}=\#\left\{\vec{u} \in \mathbb{Z}_{p}^{* d}: \kappa(\vec{u})=l\right\}, \quad \sum_{l=1}^{d} N_{d, l}=(p-1)^{d} .
$$


Hence, interchanging $\vec{x}$ and $\vec{y}$ as necessary,

$$
M=\sum_{1 \leq d, l \leq r} M_{d, l}=\sum_{1 \leq d \leq r} M_{d, d}+2 \sum_{1 \leq l<d \leq r} M_{d, l} .
$$

For a particular solution $x_{1}, \ldots, x_{r}, y_{1}, \ldots, y_{r}$ with $\kappa(\vec{x})=d, \kappa(\vec{y})=l$, we have subsets $\left\{u_{1}, \ldots, u_{d}\right\} \subseteq\left\{x_{1}, \ldots, x_{r}\right\}$ and $\left\{v_{1}, \ldots, v_{d}\right\} \subseteq\left\{y_{1}, \ldots, y_{r}\right\}$ with $D_{d}\left(u_{1}, \ldots, u_{d}\right) \neq 0$, $D_{l}\left(v_{1}, \ldots, v_{l}\right) \neq 0$. Clearly there are $\left(\begin{array}{l}r \\ d\end{array}\right)^{2}$ possibilities for $\left\{u_{1}, . ., u_{d}\right\}$, and $\left\{v_{1}, \ldots, v_{d}\right\}$, though each $\vec{x}$ will be associated with at least $(r-d+1)$ different subsets $\left\{u_{1}, \ldots u_{d}\right\}$ (since given one collection $\left\{u_{1}, \ldots, u_{d}\right\}$ with $D_{d}\left(u_{1}, \ldots, u_{d}\right) \neq 0$ we can add one of the $(r-d)$ omitted $x_{j}$ in place of an appropriate $\left.u_{i}\right)$, and likewise for the $\left\{v_{1}, \ldots, v_{d}\right\}$ if $l=d$. If $l<d$ we have at least $\left(\begin{array}{c}r-l \\ d-l\end{array}\right)+\left(\begin{array}{c}r-l \\ d-l+1\end{array}\right)=\left(\begin{array}{c}r-l+1 \\ d-l+1\end{array}\right)$ different $\left\{v_{1}, \ldots, v_{d}\right\}$ associated to a given $\vec{y}$ (since given one collection $v_{1}, \ldots, v_{l}$ with $D_{l}\left(v_{1}, \ldots, v_{l}\right) \neq 0$ we have $\left(\begin{array}{c}r-l \\ d-l\end{array}\right)$ ways to simply add an additional $(d-l)$ from the $(r-l)$ remaining $y_{i}$ and $\left(\begin{array}{c}r-l \\ d-l+1\end{array}\right)$ ways of adding $(d-l+1)$ positions from the remaining $y_{i}$ and dropping one of the $\left.v_{1}, \ldots, v_{l}\right)$. Observe that

$$
\left(\begin{array}{l}
r-l+1 \\
d-l+1
\end{array}\right) \geq \begin{cases}(r-d+1), & \text { if } d=r, \\
2(r-d+1), & \text { if } l<d \leq r-2, \text { or } d=r-1 \text { and } l<r-2, \\
\frac{3}{2}(r-d+1), & \text { if } d=r-1 \text { and } l=r-2 .\end{cases}
$$

Bound the number of values for $v_{1}, \ldots, v_{d}$ by $N_{d, l}$ and the number of $\left\{x_{1}, \ldots, x_{r}\right\} \backslash$ $\left\{u_{1}, \ldots, u_{d}\right\}$ by $(p-1)^{r-d}$. When $d<r$, once the values of $\left\{v_{1}, \ldots, v_{d}\right\}$ have been chosen, containing some subset $\left\{v_{1}, \ldots, v_{l}\right\}$ with $D_{l}\left(v_{1}, \ldots, v_{l}\right) \neq 0$, the remaining $y_{i}$ satisfy

$$
0=D_{l+1}\left(y_{i}, v_{1}, \ldots, v_{l}\right)=y_{i}^{k_{l+1}} D_{l}\left(v_{1}, \ldots, v_{l}\right)+\sum_{j=1}^{l} \lambda_{j}\left(v_{1}, \ldots, v_{l}\right) y_{i}^{k_{j}},
$$

for some polynomials $\lambda_{j}$.

If $k_{1}, \ldots, k_{l+1}$ are all of the same sign, then the degree of the resulting polynomial in $y_{i}$, and so the number of possible $y_{i}$, is bounded by $\left(\left|k_{l+1}\right|-\left|k_{1}\right|\right)<\ell_{l+1}$. Otherwise, let $k_{J}$ denote the largest magnitude exponent with $J \leq l$, opposite in sign to $k_{l+1}$. If $k_{l+1}>0$, so that $k_{J}<0$, then $k_{l+1}=\ell_{l+1}$ and $r\left|k_{J}\right|=\ell_{J} \leq \ell_{l+1}$, by (3.1). It follows that the degree of the resulting polynomial in (3.4) will be at most $\left(k_{l+1}+\left|k_{J}\right|\right) \leq(1+1 / r) \ell_{l+1}$. If $k_{l+1}<0$, then $k_{l+1}=\frac{1}{r} \ell_{l+1}$ and $k_{J}=\ell_{J} \leq \ell_{l+1}$. Thus the degree is at most $\left(\left|k_{l+1}\right|+k_{J}\right) \leq(1+1 / r) \ell_{l+1}$. Hence the number of possibilities for $\left\{y_{1}, \ldots, y_{r}\right\} \backslash\left\{v_{1}, \ldots, v_{d}\right\}$ is certainly bounded by $\left(1+\frac{1}{r}\right)^{r-d} \ell_{l+1}^{r-d}<$ $e \ell_{d+1} \cdots \ell_{r}$. Writing $\left(\begin{array}{l}r \\ d\end{array}\right)^{2}(r-d+1)^{-2}=\left(\begin{array}{c}r+1 \\ d\end{array}\right)^{2}(r+1)^{-2}$ and using (3.3) we have

$$
\begin{aligned}
M_{d, l} \leq & e \frac{\left(\begin{array}{c}
r+1 \\
d
\end{array}\right)^{2}}{(r+1)^{2}}(p-1)^{r-d} \ell_{1} \cdots \ell_{r} N_{d, l} \\
& \times \begin{cases}1, & \text { if } l=d \text { or } d=r, \\
\frac{1}{2}, & \text { if } l<d \leq r-2, \text { or } d=r-1 \text { and } l<r-2, \\
\frac{2}{3}, & \text { if } d=r-1 \text { and } l=r-2 .\end{cases}
\end{aligned}
$$

It follows from (3.2) that

$$
M \leq e(p-1)^{r} \ell_{1} \cdots \ell_{r} M_{1}(r+1)^{-2}
$$


where the factor of $e$ may be omitted if all the $k_{i}$ are positive, and

$$
M_{1} \leq \sum_{d=1}^{r}\left(\begin{array}{c}
r+1 \\
d
\end{array}\right)^{2} \frac{\sum_{l=1}^{d} N_{d, l}}{(p-1)^{d}}+\left(\begin{array}{c}
r+1 \\
r
\end{array}\right)^{2} \frac{\sum_{l=1}^{r-1} N_{r, l}}{(p-1)^{r}}+\frac{1}{3}\left(\begin{array}{c}
r+1 \\
r-1
\end{array}\right)^{2} \frac{N_{r-1, r-2}}{(p-1)^{r-1}},
$$

the second term coming from the extra contribution when $d=r$ and $l<r$ and the third term from the extra contribution when $d=r-1, l=r-2$. Now,

$$
M_{1} \leq\left(\begin{array}{c}
2 r+2 \\
r+1
\end{array}\right)-2+(r+1)^{2}+\frac{1}{12} r^{2}(r+1)^{2}<\frac{4}{r^{2}}\left(\begin{array}{c}
2 r \\
r
\end{array}\right)(r+1)^{2},
$$

and so the lemma follows. The saving of the factor of $e$ when $r=3$ can be seen by a more careful analysis of the above proof.

Proof of Lemma 3.2. For $r=2$ write $M=\sum_{\vec{u}} C(\vec{u})^{2}$ where

$$
\begin{aligned}
C\left(u_{1}, u_{2}\right) & =\#\left\{(x, y) \in \mathbb{Z}_{p}^{* 2}: x^{k_{1}}-y^{k_{1}}=u_{1}, x^{k_{2}}-y^{k_{2}}=u_{2}\right\} \\
& =d \#\left\{x \in \mathbb{Z}_{p}^{*}: x^{k_{1}}-y^{k_{1}}=u_{1}, x^{k_{2}}-y^{k_{2}}=u_{2} \text { for some } y \in \mathbb{Z}_{p}^{*}\right\}
\end{aligned}
$$

where $d=\left(k_{1}, k_{2}, p-1\right)$ (since for each $x$ with a solution there will be $d$ solutions $y$ satisfying $y^{\left(k_{1}, k_{2}\right)}=\left(x^{k_{1}}-u_{1}\right)^{s}\left(x^{k_{2}}-u_{2}\right)^{t}$ where $\left.\left(k_{1}, k_{2}\right)=k_{1} s+k_{2} t\right)$.

Clearly if $0<k_{1}<k_{2}$ are both positive, then $x$ will be a zero of the polynomial

$$
f=\left(x^{k_{1}}-u_{1}\right)^{k_{2} / d}-\left(x^{k_{2}}-u_{2}\right)^{k_{1} / d}
$$

and for $\vec{u} \neq \overrightarrow{0}$ this will be a non-zero polynomial (if $u_{1} \neq 0$, then $f$ contains a term $x^{k_{1}}$ and if $u_{1}=0$ and $u_{2} \neq 0$, a constant term) of degree (and so number of solutions) at most $\left(k_{2} / d-1\right) k_{1}$.

If $k_{1}<0<k_{2}$, then $x$ will be a root of the non-zero polynomial

$$
f=\left(x^{k_{2}}-u_{2}\right)^{\left|k_{1}\right| / d}\left(1-x^{\left|k_{1}\right|} u_{1}\right)^{k_{2} / d}-x^{\left|k_{1}\right| k_{2} / d}
$$

of degree at most $2\left|k_{1}\right| k_{2} / d$ when $\vec{u} \neq \overrightarrow{0}$.

For $u_{1}=u_{2}=0$ the number of solutions $C(\overrightarrow{0})$ to $x^{k_{1}}=y^{k_{1}}, x^{k_{2}}=y^{k_{2}}$ (and hence $\left.x^{d}=y^{d}\right)$ is $(p-1) d$. Hence, since $\sum_{\vec{u}} C(\vec{u})=(p-1)^{2}$, we have when $k_{1}$ is positive,

$$
\begin{aligned}
M & \leq k_{1}\left(k_{2}-d\right) \sum_{\vec{u} \neq \overrightarrow{0}} C(\vec{u})+d^{2}(p-1)^{2} \\
& =\left(k_{1} k_{2}-d\left(k_{1}-d\right)\right)(p-1)^{2}-d k_{1}\left(k_{2}-d\right)(p-1)<k_{1} k_{2}(p-1)^{2}
\end{aligned}
$$

and when $k_{1}$ is negative,

$$
\begin{aligned}
M & \leq 2\left|k_{1}\right| k_{2} \sum_{\vec{u} \neq \overrightarrow{0}} C(\vec{u})+d^{2}(p-1)^{2} \\
& =\left(2\left|k_{1}\right| k_{2}+d^{2}\right)(p-1)^{2}-2 d\left|k_{1}\right| k_{2}(p-1)<3\left|k_{1}\right| k_{2}(p-1)^{2} .
\end{aligned}
$$

\section{REFERENCES}

[1] N. M. Akulinichev, Estimates for rational trigonometric sums of a special type, Doklady Acad. Sci. USSR 161 (1965), 743-745. English translation in Doklady 161, no. 4 (1965), 480-482. MR $0177956(31: 2214)$

[2] F. N. Castro \& C. J. Moreno, Mixed exponential sums over finite fields, Proc. Amer. Math. Soc. 128, no. 9 (2000), 2529-2537. MR 1690978 (2000m:11070)

[3] T. Cochrane \& C. Pinner, Stepanov's method applied to binomial exponential sums, Quart. J. Math. 54, no. 3 (2003), 243-255. MR 2013138 
[4] T. Cochrane, C. Pinner \& J. Rosenhouse, Bounds on exponential sums and the polynomial Waring's problem mod p, J. London Math. Soc. (2) 67, no. 2 (2003), 319-336. MR 1956138 (2003m:11129)

[5] H. Davenport, On certain exponential sums, Journal für Math., 169 (1933), 158-176.

[6] D. R. Heath-Brown \& S. Konyagin, New bounds for Gauss sums derived from kth powers, and for Heilbronn's exponential sum, Quart. J. Math. 51 (2000), 221-235. MR 1765792 (2001h:11106)

[7] A. A. Karatsuba, On estimates of complete trigonometric sums, Matem. Zametki. 1 (1967), 199-208 (Russian); translation in Math. Notes (1968), 133-139.

[8] S. Konyagin \& I. Shparlinski, Character Sums with Exponential Functions and their Applications, Cambridge Univ. Press, Cambridge, 1999. MR 1725241 (2000h:11089)

[9] L. J. Mordell, On a sum analogous to a Gauss's sum, Quart. J. Math. 3 (1932), 161-167.

[10] G. I. Perel'muter, Estimate of a sum along an algebraic curve, Mat. Zametki 5 (1969), 373380. MR 0241424 (39:2764)

[11] I. E. Shparlinski, Computational and Algorithmic Problems in Finite Fields, Mathematics and its applications (Soviet series), Kluwer, 1992. MR 1249064 (94j:11122)

[12] A. Weil, On some exponential sums, Proc. Nat. Acad. Sci. U.S.A. 34 (1948), 204-207. MR $0027006(10: 234 \mathrm{e})$

[13] T. Wooley, A note on simultaneous congruences, J. Number Theory 58 (1996), no. 2, 288-297. MR 1393617 (97h:11037)

[14] H. B. Yu, Estimates for complete exponential sums of special types, Math. Proc. Camb. Phil. Soc. 131 (2001), 321-326. MR 1857123 (2002g:11126)

Department of Mathematics, Kansas State University, Manhattan, Kansas 66506

E-mail address: cochrane@math.ksu.edu

Department of Mathematics, Kansas State University, Manhattan, Kansas 66506

E-mail address: pinner@math.ksu.edu 